\title{
Le viol de la femme du préfet ou le récit subversif d'une inversion des rapports de domination dans les Andes
}

\section{Valérie Robin Azevedo et Camille Noûs}

\section{(2) OpenEdition}

\section{Journals}

Édition électronique

URL : https://journals.openedition.org/clo/7057

DOI : $10.4000 /$ clo.7057

ISSN : 2266-1816

Éditeur

INALCO

\section{Édition imprimée}

Date de publication : 13 août 2020

Pagination : 163-171

ISBN : 9782858313501

ISSN : 0396-891X

\section{Référence électronique}

Valérie Robin Azevedo et Camille Noûs, «Le viol de la femme du préfet ou le récit subversif d'une inversion des rapports de domination dans les Andes », Cahiers de littérature orale [En ligne], HorsSérie | 2020, mis en ligne le 02 septembre 2020, consulté le 22 mars 2022. URL : http:// journals.openedition.org/clo/7057 ; DOI : https://doi.org/10.4000/clo.7057 


\title{
Le viol de la femme du préfet ou le récit subversif $d$ 'une inversion des rapports de domination dans les Andes
}

\author{
Valérie Robin Azevedo \\ Université de Paris - URMIS
}

Camille Nô̂s

Laboratoire cogitamus

Le viol de la femme du préfet

Il y avait la femme d'un préfet. Son époux était parti au loin, au village d'Esquina.

Alors des jeunes hommes dirent :

- Je te parie que je peux me la faire, cette dame.

- Mais comment tu pourrais te la faire cette dame, toi qui n'es qu'un Indien?

- Écoute ! Eh bien moi je te parie ce que tu veux.

- Combien ?

- Je te parie un ... trois millions, quatre millions.

- OK, d'accord.

- OK, c'est bon.
Prefectopa señoranpa viulayusqanmanta

Kasqa prefectopa señoran. Hinaspas karuta wiraquchanqa rin Iskinachatas riki. Hinaspas chhayna maqt'akunas.

- Akismá chay señorata nuqa usaruyman - nispa nisqa.

Hinaspas: - Imaynatataq chay señoratari qanri usarushawaq qan indiori carajo.

- Wa! Nuqas riki imaynatapas mira apostayurusayki.

- Pero hayk'apaq.

- Ya napaq... huq... kinsa millon tawa millon aqnapaqyá.

- Ya listo.

- Ya chiqay. 
— Mais comment on va savoir si tu te l'es faite ou pas ?

- Venez demain après-midi, cachezvous derrière la maison et regardez bien. - OK, d'accord.

— D'accord, le monsieur [= le préfet] a l'habitude de se saouler et il rentrera tard, après la nuit tombée. - Alors tu ne vas pas pouvoir te la faire ? - Bon sang, mais bien sûr que je vais me la faire!

- D'accord !

Ce jeune homme emprunta alors une mantille, une jupe, un chapeau, tout ça, des habits femmes. Il se mit vite les jupons. Puis, il se remplit [le ventre] avec des chiffons, comme ça, pour ressembler à une femme enceinte. Et il se présenta [chez la femme du préfet] à la tombée de la nuit :

- Madame, je viens vous rendre visite.

— Entre, ma fille! D'où viens-tu comme ça ? - Madame, je viens d'arriver dans le coin, de passage. En fait, comme vous voyez, je suis enceinte et mes premières contractions viennent de commencer. Je me suis dit : «Que vais-je faire ? Si j'allais chez cette dame? » et je suis venue vous voir, madame. Et votre mari ? — Il est allé travailler et n'est pas encore rentré.

- Il vaut mieux que votre époux ne rentre pas. J'ai déjà fait plusieurs fausses couches et l'enfant peut se coincer [si un homme est là pour l'accouchement]. Faites-le monter de suite à l'étage [par l'escalier extérieur]. — Soit, ma fille ! — Fermez la porte à clé. Il ne faut surtout pas qu'il rentre à l'improviste. - Eh bien!
- Imaynapitaq yachasunri usasqaykita mana usasqaykita.

- Paqarin tardiyá hamunki wasin qhipamanta uyarayamuwanki. - Ya listo.

- Ya, chay wiraquchamá machayta yachan chayqa tutataña hamushanqa riki. - Bueno, manachu usarunki. - Carajo usarusaqmi! - Bueno carajo!

Chaytas chay maqt' aqa rebosata pollerata sombrerota chaykunata mañarukusqa warmi p'achata. Huqtas chay faldakunawan p'acharukun. Hinaspa thantawan ankhaynata aqnata winarukusqa wiksayuq wakirparin. Hinaspas tutayaqchatañá haykuyamun:

- Señoray visitayki. - Pasaykuy hija. Maymantaña. - Kunanmi señoray khayna hamukurani kaykunata purikuq. Ya kunan khayna unquq kapuni. Hinaspamá kunan wiksa nanay hap'iruwan. «Imaynataq kasaq. Aswan chay señorallamanña haykurusaq » nispa visitamuyki señoray... Wiraquchaykiri.

- Trabajaqmi rin, manaraq hamushanchu.

- Aman chay wiraquchaykiqa haykumunqachu. Apsukuyta yachani, chakapakuni. Hinaspan kunan altosllamanña chaskirunki. - Vaya pues, hija. - Llawiyuruy punkutaqa. Amapuni haykuyaramunqachu. - Vaya pues. 
- Aie, aïe, aïe ! Je souffre beaucoup, madame. Qu'est-ce qui va m'arriver? Je vais peut-être accoucher au beau milieu de la nuit. Qu'est-ce qui va m'arriver ? — Puisque tu es ici, je vais t'aider à accoucher.

La nuit était déjà bien avancée lorsqu'on frappa à la porte. - Monsieur Antonio arrive, [vite,] il arrive ! [dit la femme du préfet]. - Madame ! Madame ! [dit le préfet]. - Ne rentre pas. Nous avons de la visite ici. - Qui ?

- C'est une pauvre fille enceinte qui est de passage. Elle dit qu'elle a déjà fait plusieurs fausses couches, que l'enfant se coince. Aucun homme ne doit être là. Va donc à l'étage, monsieur. Je t'y apporterai ton repas. - Bon, d'accord.

- Si l'enfant se coince, elle me mourra dans les bras. - Bon d'accord, dit-il et il monta à l'étage. — Reste là-haut. Je vais t'apporter à manger dans un instant [lui dit son épouse]. — Aïe, aïe !

La femme du préfet apporta le repas [à son époux], le fit manger et revint. - Aie, aie ! Serrez-moi fort, serrezmoi fort, madame. Serrez-moi fort, serrez-moi fort. Aïe, aïe ! Vous pouvez demander, s'il vous plaît, à votre mari de me rendre un petit service ? Qu'il me joue un air rythmé au violon. S'il dit : «C'est un garçon », ça m'aidera à accoucher. Quand arrivera le moment, qu'il crie : «C ' 'est un garçon! » et qu'il joue du violon, je le paierai bien !
- Ay ay ay! nak'ariyta yachani, señora. Imaynataq kaymanri. Kushka tutakunata icha iskapakuymanchu icha, señora. Imaynataq kasaqri. - Yanqa kunanqa hamushankitaq chayayusayki.

Hinamantataqsi «tak tak tak tak allin tutataña. - Chayña wiraqucha Antonio, chayña! - Señora! Señora! - Amamá haykumunkichu. Visitanchismi kashan kaypi. $-P i$.

\section{- Huq puriq hamusqa hija unquq.} Chaysi apsukuyta yachan, chakapakuytas yachan. Chaysi mana qharita confianchu. Altosllaman ripuy wiraqucha. Chayman mikhunata apamusayki. - Vaya pues.

\section{- Chakapakuspapas}

wañuyukuwasqaypas. - Vaya pues

- siqarapun. - Aqnallapi kashanki.

Ratulla mikhunata apamusaq,

aparamusaq. - Ay ay!

Mikhunata apayuruspa mikhuyarachin. Kutiyaramun. - Ay ay! Hap'iyuway, hap'iywway, señora, hap'iyuway, hap'iyuway. Ay ay! Ya chay wiraquchaykita waliyapamuwanki. Violinta atakita tukayapamuwanqa. «Qharichan kasqa » niqtin iskaparukuymanmi. Imay oraspas chayqa «qharichan kasqa » nispa waxarikuspa atakita tukayatamuwanqa, allinllata pagapusaqmi. 
— Bon d'accord... Monsieur ! [Pablo monte le ton pour signifier que la femme du préfet doit se faire entendre de son mari]. - Madame ? - Dès qu'on t'annonce que c'est un garçon, tu te mets à lui jouer un air rythmé ... au violon. - Bon, dit-il.

[Le préfet] se saisit de son archer et s'allongea sur son lit en attendant le signal. Il était minuit, minuit était passé. S'étant bien faite serrer [le ventre], elle éloigna [la femme du préfet] : - Ah, les douleurs se sont arrêtées net, madame. — Comment ça se fait ? - Il semble que je ne vais pas encore accoucher. Je crois que le bébé a simplement changé de place, il me semble. Grâce à votre massage mes douleurs sont passées. $\mathrm{Ah}$, Madame, je vois que vous avez très sommeil et moi aussi. Allons donc nous reposer et dormir un peu, dit-elle.

- Bon, couvre-toi et [allonge-toi] là, par terre. Moi, je dormirai dans le lit. - Non. Réchauffons-nous et dormons plutôt ensemble puisque nous sommes entre femmes, dit l'autre. - Alors monte sur le lit, dit la dame en montant sur le lit.

La dame se déshabilla. L'autre aussi, dans l'obscurité ... la femme enceinte enveloppa dans sa vieille mantille ses jupes, ses robes et tous ses vêtements. Elle les mit auprès d'elle pour pouvoir s'enfuir ensuite rapidement. Puis, après ça, l'homme se glissa dans le lit.

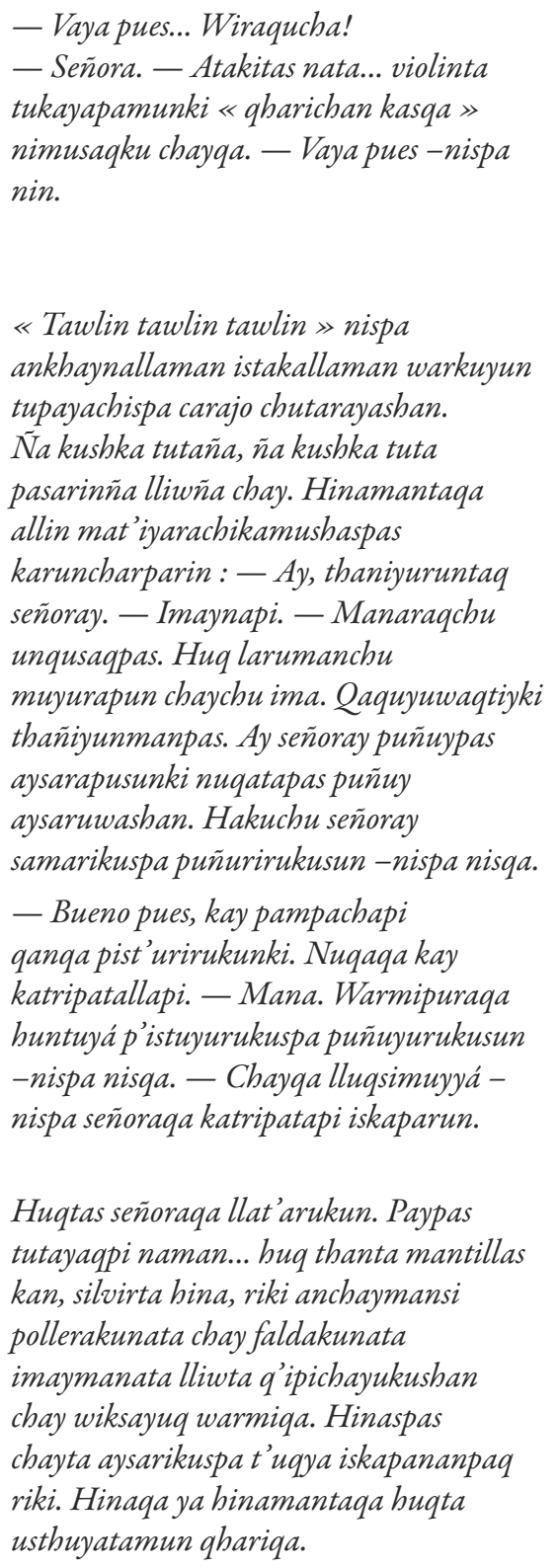

- Vaya pues... Wiraqucha! - Señora. - Atakitas nata... violinta tukayapamunki «qharichan kasqa » nimusaqku chayqa. - Vaya pues - nispa nin.

,

pas

par

.

sen

.


— Aï ! [...] La dame la repoussa franchement. [L'homme] l'attrapa. — Mais c'est un démon ce diable ! Hé ! Dors tranquillement. Qu'est-ce qui se passe ? Qu'est-ce qui se passe ? Tu es folle ou quoi ? Eh ! Eh ! Monsieur [l'épouse s'adresse à son mari] ! C'est un garçon ! C'est un garçon! Eh, eh !

Le mari était à l'étage. Alors il bondit : — Merde! Hein ? Quoi ? Ça y est, elle a accouché. Se jetant sur son violon, il se mit à jouer avec enthousiasme. Pendant ce temps, sa femme se faisait outrager. Après l'avoir violée, le salaud s'enfuit, aussi vite que l'éclair. Alors la dame sortit toute nue avec la jupe retroussée.

- Eh, démon [la femme du préfet s'adresse à son époux], je t'ai dit que c'était un garçon! Pourquoi ne m'as-tu pas écouté quand je t'appelais ? Et toi tu te mets à jouer des airs de violon ! - Bon sang, Madame, j'ai bien joué l'air [que tu m'as demandé].

— Mais non! Je suis en train de te dire que c'est un garçon, c'était un homme ! Et il vient de me violer ! - Putain de merde! Quoi ! C'est toi qui as reçu un homme là-bas dedans! Putain de merde. Et il frappa et frappa encore son épouse. Il la mit dehors toute nue, pour toujours. Depuis lors, le préfet et son épouse ne vivent plus ensemble ${ }^{1}$.
- Ay! [...] Señora franco riki wikch'urikapun i. Huqtas señorata hap'imun i carajo. - Supaytaq kay saqrari. Yaw, qasinta puñuy. Imananmi. Imananmi. Waq'ayankichu? imayna. Yaw, yaw, señor! Qharin kasqa, qharin kasqa, carajo yaw yaw...

Khayna huq laru altuspis wiraquch a kashan. Hinamantas huqtas: - Carajo! Ha! Cómo? Na chay iskaparukunñas i. Ya bigulinta aypaparikuspaqa « rikin rikin rikin rikin » nispas tukayamun. Hinaspa señoranta atakipi chaypi viulayachin. Hinaspas huqtas viulayta tukuspaqa t'uqya pasakapun qhilli runaqa. Hinaspas qhipanta faldachallantin q'alalla señoraqa iskapamun

- Yaw, saqra, « qharin kasqa » nishaykitaq! Imaqtintaq mana waxakusqayri uyarimuwankichu. Chaytaq qanri atakitaraqri tukayamunki! - Caray, señora, tukamushanitaq atakitaqa! -nispa nin.

- Manan! « Qharin kasqa » nishaykitaq, qharin kasqa. Hinaspa viulayuwan! - Carajo y mierda! Qan qharitachu chay ukhupi atindiranki. Carajo y mierda -nispa. P'anan p'anan señorata. Q'alachallata qarqurparimun hasta kunan p'unchaw. Hinaspas chaymanta ni qhariwanpas ni warmiwanpas huntuchu.

1. Il s'agit d'une version augmentée du récit quechua et de son analyse initialement publiée dans Robin Azevedo, 2008. 
Ce récit est la création de Pablo Molina, paysan quechuaphone originaire de la communauté paysanne de Lutto, province de Chumbivilcas, dans les Andes de Cuzco, au Sud du Pérou. Enregistré en décembre 1999, il est présenté ici en version bilingue quechua et français et a été traduit par Valérie Robin Azevedo. Ce récit et son protagoniste fétiche relèvent du registre des contes burlesques dont l'origine semble remonter au personnage picaresque de Pedro de Urdemalas ${ }^{2}$. Issu de la tradition orale ibérique médiévale, on en trouve la trace écrite dans plusieurs ouvrages à partir du $\mathrm{XVI}^{\mathrm{e}}$ siècle. Miguel de Cervantès a aussi contribué à le rendre célèbre dans la Comedia famosa de Pedro de Urdemalas (2015 [1610-1615]). Devenu un personnage important du folklore espagnol, il se joue des puissants auxquels il s'adresse de façon irrévérencieuse et à qui il joue de sales tours. On peut dater le transfert culturel de Pedro de Urdemalas au début de la colonisation espagnole de l'Amérique où il a fini par devenir un motif classique de la tradition orale latino-américaine. Nombre de récits incluent encore de nos jours ce genre de personnage facétieux, en se réappropriant et en remodelant la trame de l'histoire en fonction d'enjeux sociaux locaux et plus contemporains.

Le viol de la femme du préfet est un véritable vaudeville, conçu par le narrateur et ses auditeurs comme une fiction. Ce récit se base sur une inversion des rapports de domination entre Métis et Indiens, au sein d'une société agraire marquée par des rapports sociaux très hiérarchisés, hérités des structures coloniales et néocoloniales qui ont caractérisé cette région andine. Le personnage incarné par la femme dupée et violée appartient à la catégorie sociale des misti (<esp. « métis »), ce que montre l'échange entre l'épouse du préfet et la fausse femme gestante appelée hija (ma fille), terme d'adresse paternaliste souvent utilisée par les misti vis-à-vis des paysans, alors que la femme enceinte s'adresse à l'épouse du préfet en employant señora (madame). Cette dernière utilise souvent des mots espagnols dans ce récit quechua, comme pour accentuer son origine sociale car les misti s'expriment plus en espagnol que les membres des communautés paysannes. Qui plus est, faire dormir par terre une femme sur le point d'accoucher vient souligner l'infériorité de son statut, au bas de la hiérarchie sociale, par rapport à son hôtesse. Cette scène reflète la banalisation des rapports de sujétion existant autrefois dans cette province, tel le fait de faire dormir une Indienne enceinte à même le sol, comme on le ferait avec une chienne qui va mettre bas. Par ailleurs, le mot maqt'a, pour désigner les garçons qui discutent du pari au début du récit, montre qu'il s'agit d'adolescents issus d'une communauté paysanne. De même,

2. Nous remercions César Itier pour nous avoir indiqué la genèse de cette tradition littéraire autour de Pedro de Urdemalas. 
le terme indio [Indien], employé par le narrateur pour se référer au principal protagoniste, et l'insistance de ses amis sur cette identification comme Indien, ne sont pas anodins dans ce contexte. D’ordinaire, Pablo Molina évoquait campesino [paysans] ou comunero pour évoquer les habitants des communautés. Indio est un terme péjoratif, utilisé de nos jours comme insulte ou pour illustrer les rapports de domination entre groupes sociaux. L'hétéro-désignation d'Indien reste en effet associée à l'époque antérieure à la Réforme agraire et à l'état de quasi-servage de certains paysans sans terre vis-à-vis des propriétaires terriens abusifs [gamonales]. Rappeler au protagoniste sa condition d'Indien vient rappeler sa place subordonnée par rapport au préfet et son épouse.

Une fois la position hiérarchique de chaque protagoniste établie, l'inversion du comportement communément associé aux paysans et aux misti se met en place. En effet, c'est la femme enceinte, donc le paysan indien, qui mène le jeu et reçoit satisfaction à ses exigences, ce qui lui accorde une position de pouvoir exceptionnelle. Préfet et épouse sont ridiculisés par un comique de situation qui s'intensifie au fil de la narration, par le biais du jeu de mots, peu perceptible dans la traduction, autour de l'expression qharin kasqa [c'est un garçon/c'est un homme]. Le terme qhari désigne la gent masculine en général. Il peut être utilisé pour parler d'un homme adulte comme pour indiquer le sexe d'un nouveau-né en quechua. Abusé jusqu'à la fin du récit, le préfet ne porte pas secours à son épouse. Pire, il accompagne le forfait d'un air de musique rythmé. Sa femme doit insister pour qu'il comprenne enfin la supercherie et le viol commis. Ce récit met ainsi en exergue la naïveté et la bêtise du préfet et de sa femme auxquelles font pendant l'intelligence et l'astuce du jeune homme.

La narration de cette parodie suscita les fous rire de l'auditoire. Certains s'étonneront qu'on s'esclaffe d'une histoire de viol. Cependant, nul ne doutait du caractère improbable de cet événement. Que comprendre de cette hilarité ? Le protagoniste indien assume un rôle peu réaliste, ce que les portraits sciemment caricaturaux des trois personnages et le stratagème rocambolesque laissent aussi sous-entendre. L'inversion des rapports de domination constitue le motif central de cette histoire. Par la ruse, la fausse femme enceinte subvertit les rapports habituels entre misti et Indiens. Il est significatif que le moyen choisi pour incarner cette inversion soit précisément le viol. Je n'ai pas eu connaissance de viols de femmes misti par des paysans dans cette région. Et si de tels actes avaient été perpétrés, l'époux de la femme abusée ne se serait probablement pas limité à la frapper et à la répudier. Le paysan violeur aurait certainement été recherché et puni. Ici, l'humiliation subie et la honte du préfet l'empêchent de venger l'outrage qui s'est déroulé presque sous ses yeux. En revanche, le parallèle avec la situation opposée, celle vécue autrefois par les habitants des communautés, est frappant. 
À Chumbivilcas, avant la Réforme agraire, les violences sexuelles étaient une pratique courante des gamonales, qui pratiquaient un droit de cuissage assumé à l'encontre des Indiennes.

Analysant l'impact de la colonisation au sein des sociétés africaines, Georges Balandier (1971, p. 202-214) soulignait que toute administration coloniale s'efforce d'expulser, ou du moins de geler, le politique des manifestations de la vie indigène. Cette entreprise de stérilisation de l'activité politique impliqua souvent un phénomène de transfert et les réactions à la domination sont parfois intervenues là où on ne les attendait pas. Les problèmes posés aux sociétés du fait de la situation coloniale se sont parfois traduits par le remaniement de thèmes qui se sont exprimés dans le langage des mythes. Si les Andes ne sont plus en situation coloniale stricto sensu, les rapports de domination et de dépendance historiques entre Indiens et métis ont caractérisé l'histoire de la société chumbivilcana jusqu'aux années 1970. La mise en scène du viol procède à un renversement imaginaire de la violence et des formes d'assujettissement auquel étaient de fait soumis les paysans. Ce texte nous plonge donc au cœur de «l'infrapolitique des dominés » évoquée par James Scott (2008) lorsqu'il propose d'éclairer les liens entre discours et relations de pouvoir. Il s'intéresse au « texte caché » et au « petit théâtre » des dominés qui élaborent des formes discursives de résistance à la domination pour maquiller insubordination et critique du pouvoir. Cet « art du déguisement politique » se matérialise avec une grande force narrative dans ce récit d'inversion fantasmée.

À travers la création littéraire, et le recours à l'humour et à l'ironie, Pablo Molina exprime une critique acerbe des anciennes formes d'exploitation pratiquées par les puissants dans ces campagnes andines qui continuent de marquer la mémoire collective régionale. Derrière ces éclats de rire partagés et l'inventivité à l'œuvre dans cette fiction s'exprime une accusation implicite des rapports de domination des Métis sur les Indiens ; en particulier les viols des femmes indiennes que les hommes des communautés, à l'instar du préfet dans le récit présenté, n'eurent guère la possibilité de contrer frontalement.

\section{Bibliographie}

Balandier Georges, 1967, Sens et puissance, Presses universitaires de Fance, Paris, $334 \mathrm{p}$.

Cervantès Miguel (de), 2015 [1610-1615], Pedro de Urdemalas, en Comedias y Tragedias, ed. Adrián J. Sáez, Real Academia Española, Madrid, pp. 795-906. 
LE VIOL DE LA FEMME DU PRÉFET OU LE RÉCIT SUBVERSIF D'UNE INVERSION DES RAPPORTS DE DOMINATION DANS LES ANDES Valérie Robin Azevedo \& Camille Noûs

Robin Azevedo Valérie, 2008, Miroirs de l'Autre vie. Pratiques rituelles et discours sur les morts dans les Andes de Cuzco, Société d'ethnologie, Nanterre, $328 \mathrm{p}$.

Scott James, 2008 [1992], La Domination et les arts de la résistance. Fragments du discours subalterne, Éditions Amsterdam, Paris, $270 \mathrm{p}$. 